\title{
Energy Method Solution for the Vertical Deformation of Longitudinally Coupled Prefabricated Slab Track
}

\author{
Juanjuan Ren, ${ }^{1,2}$ Shijie Deng, ${ }^{1,2}$ Zhibin Jin, ${ }^{1,2}$ Junbin Yang, ${ }^{1,2}$ and Xueyi Liu ${ }^{1,2}$ \\ ${ }^{1}$ MOE Key Laboratory of High-Speed Railway Engineering, Southwest Jiaotong University, Chengdu 610031, China \\ ${ }^{2}$ School of Civil Engineering, Southwest Jiaotong University, Chengdu 610031, China \\ Correspondence should be addressed to Juanjuan Ren; renjuanjuan1983@hotmail.com and Zhibin Jin; jinzhibin1979@163.com
}

Received 26 September 2016; Revised 6 February 2017; Accepted 15 February 2017; Published 20 March 2017

Academic Editor: Roman Lewandowski

Copyright (c) 2017 Juanjuan Ren et al. This is an open access article distributed under the Creative Commons Attribution License, which permits unrestricted use, distribution, and reproduction in any medium, provided the original work is properly cited.

\begin{abstract}
Upwarping on the longitudinally coupled prefabricated slab track system caused by the rising temperature is a common distress, which deteriorates the mechanical properties of the coupled slabs and the vertical stability of slabs. The objective of this paper is to quantify the upwarping deformation on the slab subjected to temperature force and to find out the influence of different factors on the upwarping phenomenon of the slabs. An analytical expression is deduced using energy method, and a finite element model is also established to verify the analytical solution's adequacy. The following main findings are drawn: (1) when the amplitude of the initial elastic misalignment $w_{e}$ is equal to a half of the amplitude of the initial plastic misalignment $w_{p}$ and the half-wavelength $l_{\min }$ takes the most unfavorable value, the maximum relative error between the analytical solution and the result in FEM is only $2.64 \%$, which demonstrates that the analytical solution correlates well with the FEM results. (2) $l_{\min }$ is closely related with $w_{p}$. With the increase of $w_{p}, l_{\min }$ becomes longer, and the maximum length of the half-wavelength is $7.769 \mathrm{~m}$. (3) When the total amplitude $w_{e}+w_{p}$ exaggerates, the slab will be much likely to suffer upwarping.
\end{abstract}

\section{Introduction}

Since slab track is characterized by its high stability, availability, reliability, and low maintenance associated costs, it has been a developing trend for high-speed railways [1]. By September in 2016, China has completed the construction of $20,000 \mathrm{~km}$ high-speed railways among which the slab track technology accounts for about $78 \%$. The longitudinally coupled prefabricated slab track (LCPST) which is reinnovated from the prototype of Bögl slab of Germany by China has been widely used on high-speed railway or Passenger Dedicated Lines (PDLs) in China with the total length of about $4637 \mathrm{~km}$ (double track). In 2008, this kind of track was firstly put into operation on the Beijing-Tianjin intercity PDL and gradually popularized on Beijing-Shanghai High-Speed Railway, Shanghai-Hangzhou Railway, Shijiazhuang-Wuhan PDL, and so forth in the following years [1]. So far, the first LCPST has been served for almost 9 years in China.

The LCPST design uses prefabricated concrete slab made of reinforced concrete which is $0.2 \mathrm{~m}$ thick, $2.55 \mathrm{~m}$ wide, and
$6.45 \mathrm{~m}$ long. The main components of the LCPST system include rail $\mathrm{CN} 60$ (Chinese standard rail with rail mass per meter $60 \mathrm{~kg} / \mathrm{m}$ ), WJ-8 type of elastic fastening system invented in China, prefabricated slabs, cement asphalt mortar (CA mortar), and hydraulic material layer, which are displayed in Figure 1. In this system, breaking points are notched every $650 \mathrm{~mm}$ at the face of the slab to form dummy joints. The threaded steels of the prefabricated slab are coupled in a longitudinal direction by tumbuckles [2]; after that sealing concrete is cast into the space between the slabs to form broad joint and narrow joint as shown in Figure 2. The width of a broad joint is $0.2 \mathrm{~m}$. The weight of a slab is about $9 \mathrm{t}$ (without rail fastening and rails).

As can been seen, the LCPST stands out its advantages not only in mechanical and structural aspects but also in technology's. For instance, preferable continuity or integrity is insured due to tumbuckles. Rail ditches are processed with the help of Computer Numeric Control (CNC) grinder with high precision to guarantee the accurate adjustment [1]. Exact level and alignment are measured efficiently owing to an 


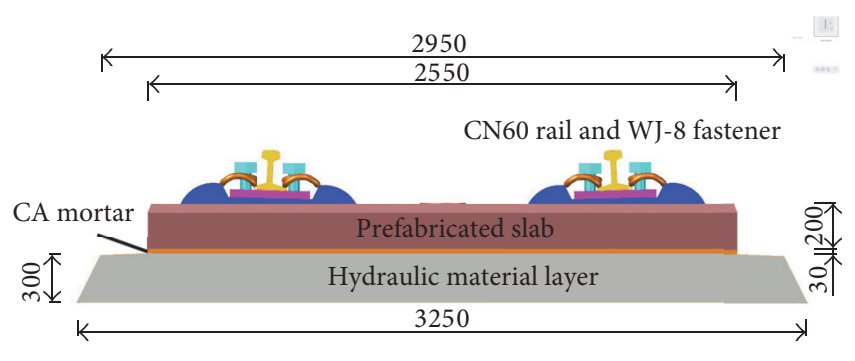

FIgURE 1: The cross section of LCPST (unit: mm).

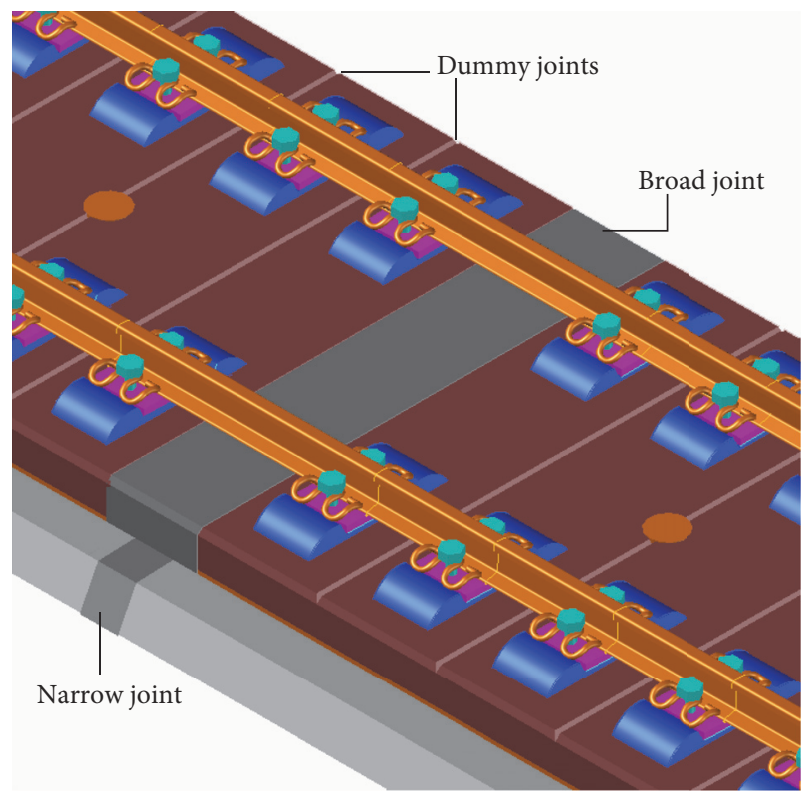

FIGURE 2: Dummy joints, broad joint, and narrow joint in LCPST.

advanced adjustment device and there is a same structural type of LCPST at subgrade, bridge, and tunnel. Nevertheless, external factors such as differential drying shrinkage and creep of the material and temperature contribute a straight slab to initial upward curl. The slab and CA mortar of LCPST are bonded together due to the bonding behavior of CA mortar. However, this kind of bonding is deteriorated from the long-term operation; therefore the longitudinal forces in the coupled slabs caused by an increase of temperature may result in irrevocable loss of bonding strength. This loss leads to diminished mechanical performance between the slab and CA mortar, and sometimes forms debonding, which is displayed in Figure 3. Because the concrete at broad joint and the coupled slab are not cast in synchrony, a weak location can be found, and then cracking at this place is most likely to occur. If slabs with initial misalignments experience higher temperature, the top surface of slabs will elongate, while the bottom will compress, causing coupled slabs to a potential upwarping, which is demonstrated as a practical problem in LCPST. In the summer of 2013, surveys about upwarping at Beijing-Shanghai PDL and Ningbo-Hangzhou High-Speed Railway have been done by the research team of the author from Southwest Jiaotong University; both

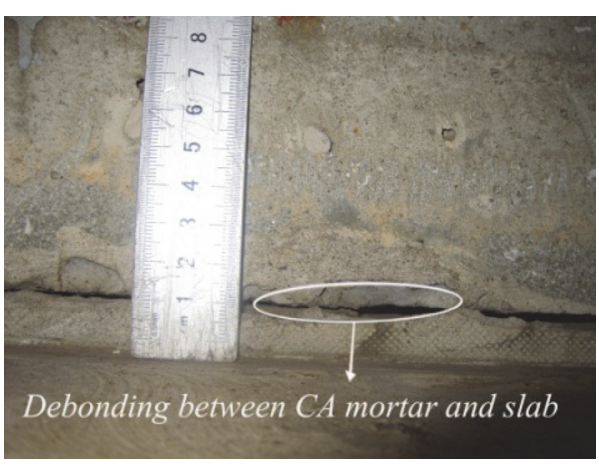

FIGURE 3: Debonding between CA mortar and coupled slab.

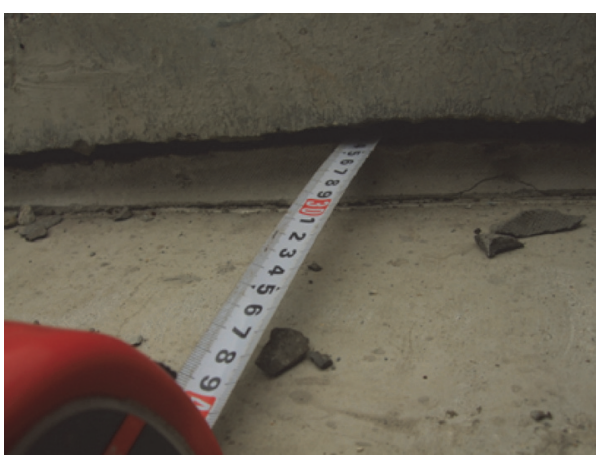

FIGURE 4: Slab upwarping on Ningbo-Hangzhou High-Speed Railway.

showed that upwarping of slab occurred preferably around broad joint. Upwarping of coupled slab at bridge at BeijingShanghai PDL was measured to be approximately $5 \mathrm{~mm}$ in total from its initial configuration, whereas it was about $20 \mathrm{~mm}$ at $\mathrm{K} 170 \mathrm{~km}+545 \mathrm{~m}$ (the mileage of this position) of Ningbo-Hangzhou High-Speed Railway (see Figure 4). Slab upwarping not only may influence the geometric alignment of railway and the ride comfort but also brings about potential danger to the bearing capacity of track structure. Thus, research on this particular topic is required.

Some analytical models have been developed to study upwarping of the coupled slab. Ren et al. preliminary analyzed the causes of upwarping of continuously reinforced concrete slabs for a twin-block slab track on the subgradebridge transition section and sorted out a repair scheme for upwarping of track slab through data analysis [3]; Liu and Feng put forward the repair measure for upwarping defect of LCPST [4]; Liu el al. analyzed the upwarping laws and influence factors of LCPST under the construction [5]; Lin et al. analyzed the nonlinear compressive stability of LCPST by taking the upwarping deformation of slab under a train load as the initial bending defects [6]. Longitudinal forces of coupled slabs induced by temperature are the most significant factor. Zhang et al. studied the influence of changeable temperature on mortar layer's relative displacement with track slab and put forward the reasonable construction temperature for LCPST [7]; Xu and Zhang established a nonlinear finite space mechanical model for calculating the 


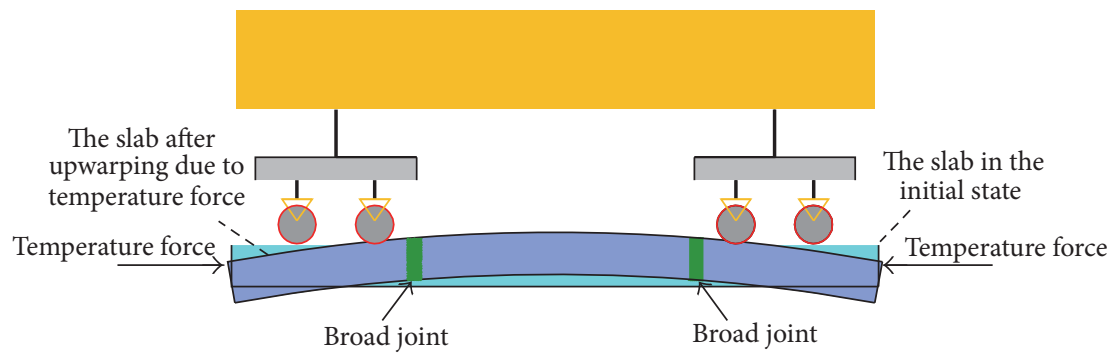

FIGURE 5: Flexural behavior of the coupled slabs model.

longitudinal force of Bögl longitudinal connected ballastless track on high-speed railway bridge [8].

Previous mentioned research work mostly covered FEM and experiment; the analytical solution has been rarely applied to study on the upwarping deformation of LCPST, but similar problem on the buckling of Continuous Welded Rail track has been investigated a lot using the analytical solution. The main difference between models of upwarping of slab track and buckling of Continuous Welded Rail (CWR) consists in different component of potential energy; nevertheless the analysis methods hold many similarities. A rise of temperature can generate compressive stresses in the rails in CWR tracks. Since the longitudinal expansion of the rails is constrained, generated compressive stresses may cause track buckling in the horizontal plane. Early in 1978, Kerr has presented a beam model to calculate the buckling load of CWR [9]. Martínez et al. present an analytical model to obtain the buckling load of a CWR track [10]. Luo et al. studied the relationship between the rail's longitudinal temperature force and the natural frequency [11]. Manap has explored the changes of longitudinal stress distribution in the rails due to mechanical loading applied by a travelling train [12]. The stability equation of CWR track (unified formula for short) in China has been derived since the 1970s based on the principle of stationary potential energy [13]. And the equation which has been compiled in the Code for Design of Railway Continuous Welded Rail [14] works well in practical applications.

Unlike the previously developed studies on the upwarping of LCPST, which mainly rely on FEM or experiment, this paper is based on the minimization of potential energy and takes coupled slabs with initial misalignments into consideration. The analytical expression is obtained based on the energy method.

\section{Methodology}

The main difference between models of upwarping of coupled slabs and buckling of CWR consists in different components of the total potential. However, the analysis method and the initial state hold many similarities. The slab under study is slender; therefore, it can be modeled as an Euler-Bernoulli beam. Both models share the similar flexural behavior when both are subjected to temperature forces in the longitudinal direction. The flexural behavior of the coupled slabs model is shown in Figure 5.

2.1. Theoretical Background. Unified formula has been applied to analyze the stability of Continuous Welded Rail, and it works well in practice. It is assumed that the coupled slabs are considered as a beam with two initial misalignments (the initial elastic misalignment and the initial plastic misalignment), which share the same wavelength with the length of the coupled slabs, lying on the homogeneous medium. During the buckling process, the compression deformation energy of rail, deformation energy of track frame, and transverse deformation energy of ballast bed were calculated, respectively [15]. Applying the principle of stationary potential energy, the unified formula was released by MOR (the former Ministry of Railway of China) in 1977 [13]. As for the coupled slab, the hypotheses are the same as the rail's previously mentioned. The upwarping load of compressed slabs is the one for which the slabs find themselves in a neutral equilibrium state. For this reason, according to the energy method, the value of the upwarping load can be obtained from the system's total potential $W$, which is a function of the generalized coordinates of the structural system.

2.2. Model Assumptions. While the debonding of CA mortar occurs, CA mortar does not work well and has terrible bonding behavior, so that the coupled slab and CA mortar are separated. In this paper, coupled slabs are solely focused on, and the effect of CA mortar is ignored. For simplicity, there are some assumptions which are given as follows:

(a) The effect of rail, fastening system is all neglected.

(b) During the upwarping process, it is assumed that the ends of the slabs remain where they stay, with hinge support at both ends.

(c) It is assumed that coupled slabs and CA mortar are completely separated in the debonding zone.

(d) The chord length $l$ is assumed to be unchanged during the process of upwarping.

(e) The influence of adjacent broad joints is not taken into account in the analysis. 


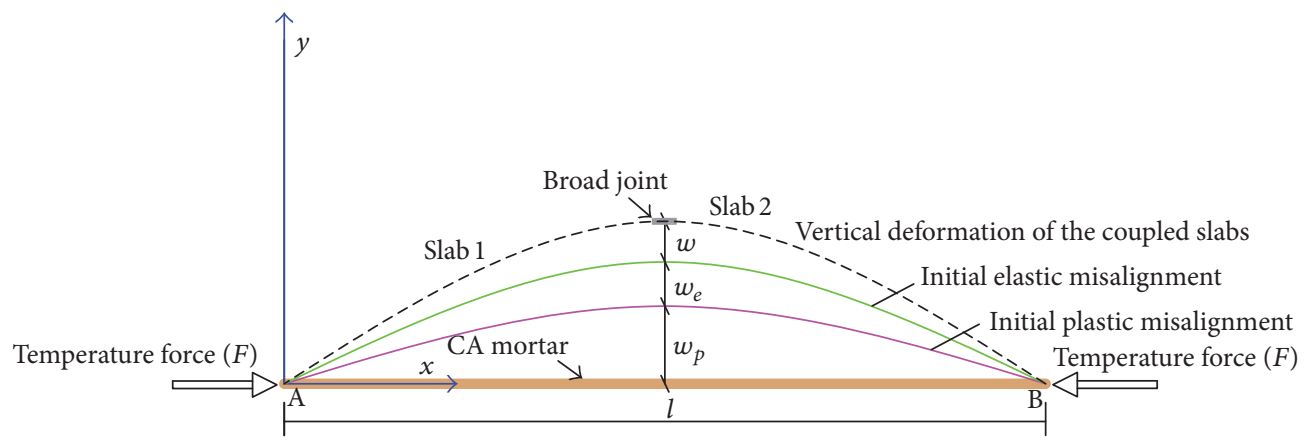

FIGURE 6: Upwarping deformation of the coupled slabs.

(f) The stiffness of a broad joint is considered as a reduction of the stiffness of the coupled slab.

(g) The concept of the initial misalignments is similar to that in the model of buckling; both include an initial elastic misalignment and an initial plastic misalignment and share the same wavelength with the beam.

Just as previously mentioned, coupled slabs can be simulated as an equivalent beam, with the same cross section and inertia as a real slab. Surveys at Beijing-Shanghai PDL and Ningbo-Hangzhou High-Speed Railway revealed that upwarping of the coupled slabs occurred preferably around broad joint, where there is a stiffness difference between the prefabricated slab concrete and the cast in-site concrete, so the upwarping zone ranges from $\mathrm{A}$ to $\mathrm{B}$ in Figure 6. It is hypothesized that, during the upwarping process, the coupled slabs will deform by amplifying the initial misalignments (see Figure 6). Upwarping of slabs holds similar behaviors to buckling of CWR, especially in the regularity of deformation. For the buckling model, the beam is loaded by axial compressive forces and is elastically supported by laterally distributed resistance which is proportional to the lateral deformation. While the compressive forces develop, the beam will deform by amplifying the initial misalignments. For the upwarping model, the beam is loaded by axial compressive forces and is subject to a vertical distributed resistance (dead weight) which is proportional to the vertical deformation, and the deformation form is similar to that of buckling. In [16], Liu and Guang compared different types of deformation curve in the unified formula, and the result of calculation shows that sinusoidal curve is the most similar to the conclusions from German railway, so sinusoidal curve is used to calculate the stability of CWR. Also, the vertical deformation of the coupled slabs $y_{f}$ under the action of the compressive temperature force is assumed to be a sinusoidal curve with the following equation:

$$
y_{f}=w \cdot \sin \frac{\pi x}{l}
$$

where $w$ denotes the amplitude of the upwarping deformation and $l$ denotes the half-wavelength of the slabs, just as illustrated in Figure 6.
Under the effect of temperature, the misalignment shown in Figure 6 will occur on the slabs. The initial elastic misalignment $y_{e}$ is taken as

$$
y_{e}=w_{e} \cdot \sin \frac{\pi x}{l},
$$

where $w_{e}$ denotes the amplitude of the initial elastic misalignment.

The coupled effect of the manufacture error, creep, and thermal expansion of concrete will cause unrecovered deformation of the slab, which results in an initial plastic misalignment. With the amplitude $w_{p}$, the expression of the initial plastic misalignment $y_{p}$ is

$$
y_{p}=w_{p} \cdot \sin \frac{\pi x}{l} .
$$

The function of vertical curve is to provide a gradual transition from one grade to another for smooth riding. Vertical curve represents one kind of initial misalignments. We can find in [17] that the arc is assigned to use in high-speed railway design in China. Since the sagitta of vertical curve is an irrational expression and is difficult for integration, an approximate equation is taken instead. With the radius of vertical curve $R$, the initial plastic misalignment $\left(y_{R p}\right)$ could be expressed as

$$
y_{R p}=\frac{(l-x) l}{2 R} .
$$

Thus, taking the initial elastic and plastic misalignments of the coupled slabs into consideration, the total deformation curves of LCPST with temperature force $\left(y_{F \text { total }}\right)$ or without temperature force $\left(y_{0 \text { total }}\right)$ are, respectively,

$$
\begin{aligned}
y_{\text {Ftotal }}= & y_{f}+y_{e}+y_{p}+y_{R p} \\
= & w \cdot \sin \frac{\pi x}{l}+w_{e} \cdot \sin \frac{\pi x}{l}+w_{p} \cdot \sin \frac{\pi x}{l} \\
& +\frac{(l-x) l}{2 R} \\
= & \left(w+w_{e}+w_{p}\right) \sin \frac{\pi x}{l}+\frac{(l-x) l}{2 R}, \\
y_{0 \text { total }}= & y_{e}+y_{p}+y_{R p}=\left(w_{e}+w_{p}\right) \sin \frac{\pi x}{l}+\frac{(l-x) l}{2 R} .
\end{aligned}
$$




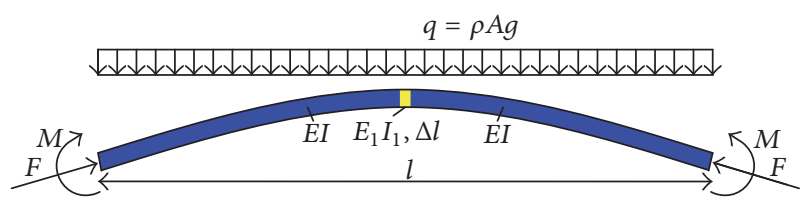

FIgURE 7: Equilibrium of axial compression $F$, gravity $q$, and bending moment $M$ during upwarping.

2.3. Model Description. Considering all the hypotheses previously mentioned, the model can be accomplished using a compressive temperature load $F$ which is applied at both ends of the misalignment and causes additional bending moment $M$ to resist the upwarping. The beam (the blue one in Figure 7) with consideration of its gravity in the calculated model, which represents the coupled slab, has a half-wavelength $l$, a uniform cross section area $A$, a moment of inertia $I$, and a modulus of elasticity $E$. Besides, we simulate the broad joint using the second beam (the yellow one in Figure 7), which has a length $\Delta l(\Delta l=0.2 \mathrm{~m})$, a same cross section area as the coupled slab, a moment of inertia $I_{1}$, and a modulus of elasticity $E_{1}$. And $q$ denotes the force per unit length generated by the gravity of coupled slabs.

The total potential energy $W$ of the model is the sum of the deformation energy associated with the compressing the slabs $W_{1}$, the energy associated with bending the slabs $W_{2}$, and gravitational potential energy of slabs $W_{3}$. According to the previous assumptions, the parameters that describe the deformed shape of the structural system are the halfwavelength of the slabs $l$ and the amplitude of the upwarping deformation $w$. The total potential energy of the model based on principle of minimum stationary potential energy [13] is obtained by

$$
\begin{aligned}
& d W=d W_{1}+d W_{2}+d W_{3}=0 \\
& d W=\frac{\partial W}{\partial w} \delta w+\frac{\partial W}{\partial l} \delta l=0 .
\end{aligned}
$$

Since assumption (d) is given, that is, $\delta l=0$, hence the equilibrium equation can be written as

$$
d W=\frac{\partial W_{1}}{\partial w}+\frac{\partial W_{2}}{\partial w}+\frac{\partial W_{3}}{\partial w}=0
$$

2.3.1. The Deformation Energy Associated with Compressing the Slabs $W_{1}$. Once the model is subject to axial compression, the compression deformation energy of the slabs can be expressed as

$$
W_{1}=F \cdot \Delta \text {. }
$$

The parameter $\Delta$ is the change in the arc length (not the chord length) of the coupled slabs, $\Delta=\Delta l_{F}-\Delta l_{0}$, in which $\Delta l_{F}$ represents arc-chord difference after deformation

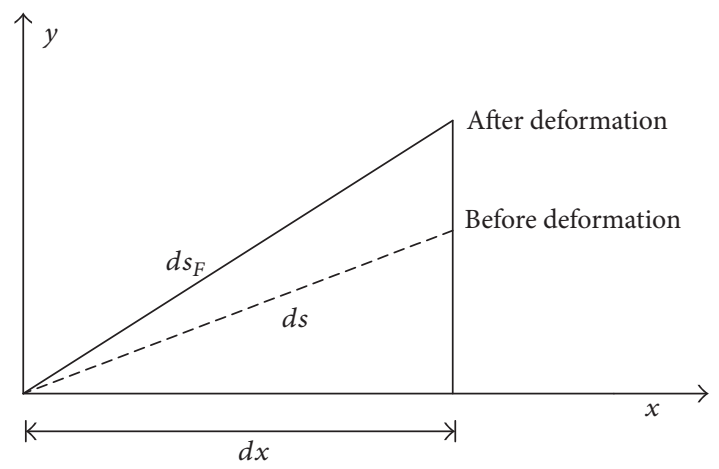

Figure 8: Arch-chord difference of the coupled slabs before and after deformation.

and $\Delta l_{0}$ represents arc-chord difference before deformation. According to Figure 8, we have

$$
\begin{aligned}
& \Delta l_{F}=\int_{0}^{l} d s_{F}-\int_{0}^{l} d x, \\
& d s_{F}=\sqrt{1+\left(y_{F \text { total }}^{\prime}\right)^{2}} d x,
\end{aligned}
$$

in which $d s_{F}$ is arc-differential after deformation.

Using a binomial series expansion for the quadratic terms and neglecting higher-order terms, we have

$$
d s_{F}=\frac{1}{2}\left(y_{F \text { total }}^{\prime}\right)^{2} d x .
$$

Similarly,

$$
d s_{0}=\frac{1}{2}\left(y_{0 \text { total }}^{\prime}\right)^{2} d x
$$

where $d s_{0}$ is arc-differential before deformation.

Integrating the difference $d s_{F}-d s_{0}$ over the beam's span, the resulting deformation $\Delta$ is

$$
\Delta=\int_{0}^{l}\left(\left(y_{\text {Ftotal }}^{\prime}\right)^{2}-\left(y_{\text {Ototal }}^{\prime}\right)^{2}\right) d x .
$$

The energy becomes

$$
\begin{aligned}
W_{1} & =\frac{F}{2} \int_{0}^{l}\left(\left(y_{F \text { total }}^{\prime}\right)^{2}-\left(y_{\text {Ototal }}^{\prime}\right)^{2}\right) d x, \\
\frac{\partial W_{1}}{\partial w} & =-\frac{F}{2} \cdot \frac{\pi^{2}\left(w+w_{e}+w_{p}\right)}{l}-\frac{2 F w l}{\pi R} .
\end{aligned}
$$

2.3.2. The Energy Associated with Bending the Slabs $W_{2}$. During the upwarping process of coupled slabs, deformation energy could be stored inside and can be written as $(1 / 2) \int M d \theta$, where $\theta$ is the angle of rotation induced by the upwarping of the slabs [15]. The energy associated with bending the slabs is composed of two parts, first of which is the energy of deformation $\int_{0}^{l} M_{e} d \theta$, stored in the beam because of the deformation caused by $M_{e}$ (the internal 
TABLE 1: The tentative calculation about the damage of the broad joint/radius of the slab.

\begin{tabular}{lccccccccc}
\hline Number & $R / \mathrm{m}$ & $l / \mathrm{m}$ & $E I / \mathrm{kN} \cdot \mathrm{m}^{2}$ & $E_{1} I_{1}$ & $w_{e} / \mathrm{m}$ & $w_{p} / \mathrm{m}$ & $w / \mathrm{m}$ & $F / \mathrm{kN}$ & The contribution rate of $E_{1} I_{1} / R$ to $F$ \\
\hline 1 & $\infty$ & & & $1 E I$ & 0.003 & 0.006 & 0.003 & 12724 & \\
2 & $\infty$ & 6.45 & 60350 & $0.5 E I$ & 0.003 & 0.006 & 0.003 & 12508 & $1.7 \%$ \\
3 & 20000 & & & $1 E I$ & 0.003 & 0.006 & 0.003 & 12701 & $1.78 \%$ \\
\hline
\end{tabular}

bending moment of the initial elastic misalignment), while the other is the energy of deformation $(1 / 2) \int_{0}^{l} M_{f} d \theta$, stored due to the increase of the internal bending moment $M_{f}$ during the process of upwarping. Hence,

$$
W_{2}=\frac{1}{2} \int_{0}^{l} M_{f} d \theta+\int_{0}^{l} M_{e} d \theta
$$

where $d \theta=y_{f}^{\prime \prime} d x, M_{f}=K E I y_{f}^{\prime \prime}$, and $M_{e}=K E I\left(y_{0}-y_{p}-\right.$ $\left.y_{R}\right)^{\prime \prime}$.

EI represents flexural rigidity of the slab (EI will be replaced by $E_{1} I_{1}$ at the zone of the broad joint) and $K$ represents the reduction factor of flexural rigidity of track slab due to the influence of dummy joints, and the formula is obtained:

$$
\begin{aligned}
\frac{\partial W_{2}}{\partial w}= & \frac{K E_{1} I_{1} \pi^{3}\left(w+w_{e}\right)(\pi \Delta l+l \sin (\pi \Delta l / l))}{2 l^{4}} \\
& -\frac{K E I \pi^{3}\left(w+w_{e}\right)(\pi \Delta l+l \sin (\pi \Delta l / l)-\pi l)}{2 l^{4}} .
\end{aligned}
$$

2.3.3. Gravitational Potential Energy of Slab $W_{3}$. As mentioned in Section 2.3, the gravity of the slabs is assumed to be the distribution force along the longitudinal direction of slabs. The gravitational potential energy of track slab could be expressed by

$$
\begin{aligned}
W_{3} & =\int_{0}^{l} \int_{0}^{y_{f}} \rho A g d y_{f} d x, \\
\frac{\partial W_{3}}{\partial w} & =\rho A g \times \frac{2 l}{\pi},
\end{aligned}
$$

where $\rho$ stands for the density of the slab: $\rho=2500 \mathrm{~kg} / \mathrm{m}^{3}$. $A$ is the area of the cross section, $A=0.51 \mathrm{~m}^{2}$, and $g$ is gravitational acceleration: $g=9.8 \mathrm{~m} / \mathrm{s}^{2}$.

2.4. Analytical Solution. Substituting (14), (16), and (18) into (7), the calculation formula of temperature force is

$$
F=\frac{K\left(E_{1} I_{1}-E I\right)(\pi \Delta l+l \sin (\pi \Delta l / l)) \pi^{4}\left(w+w_{e}\right) / 4 l^{5}+K E I \pi^{5}\left(w+w_{e}\right) / 4 l^{4}+\rho A g}{\pi^{3}\left(w+w_{e}+w_{p}\right) / 4 l^{2}+1 / R}
$$

According to Code for Design of High Speed Railway (TB 10621-2014) [18], $20000 \mathrm{~m}$ or $25000 \mathrm{~m}$ is selected as the radius of vertical curve depending on the speed of passenger train. Considering the most unfavorable condition, we choose $20000 \mathrm{~m}$. We take $l$ as the length of a slab. Because the slab is made of concrete C55 (concrete grade), the elastic modulus of the slab is $35500 \mathrm{MPa}$. The cross section of the slab is a $2.55 \mathrm{~m}$ $\times 0.02 \mathrm{~m}$ rectangle, so the inertia of the slab is $1.7 \times 10^{-6} \mathrm{~m}^{4}$. $E I=35500 \times 10^{6} \times 1.7 \times 10^{-6}=60350 \mathrm{kN} \cdot \mathrm{m}^{2}$. Based on assumption ( $f$ ) in the second section of the second chapter, Model Assumptions, we take half stiffness of the slab as the stiffness of broad joint $\left(E_{1} I_{1}=0.5 E I\right)$.

Table 1 shows that the damage of the broad joint $(0.5 E I)$ influences just $1.7 \%$ of the total temperature force compared with the nondamage of the broad joint. Also the radius of the vertical curve $(R=20000 \mathrm{~m})$ influences just $1.78 \%$ of the total temperature force compared to the slab without the vertical curve $(R=\infty)$.

It could be determined from tentative calculation that the damage of the broad joint and the vertical curve provides less contribution to the temperature force. So they could be ignored in the following verification procedure, and the formula could be simplified as follows:

$$
F=\frac{K E I \pi^{5}\left(\omega+\omega_{e}\right) / 4 l^{4}+\rho A g}{\pi^{3}\left(\omega+\omega_{e}+\omega_{p}\right) / 4 l^{2}} .
$$

It could be obtained from (20) that $F$ is closely related to half-wavelength $(l)$, amplitude of the initial elastic misalignment $\left(w_{e}\right)$ and amplitude of the initial plastic misalignment $\left(w_{p}\right)$, amplitude of the deformation $(w)$, bending rigidity of the track slab EI, and dead weight of track slab within the half-wavelength range. However, parameters such as $w_{e}$ and $w_{p}$ are hard to be ascertained. To solve this problem, we take the stationary value of the compression into account.

Since $w$ is a function of the compression $F$ and $l$ is a parameter representing the half-wavelength, (20) represents a functional relationship among the axial compression, the upwarping half-wavelength, and the deformation of the slabs. From (20), it can be found that when the amplitude of 


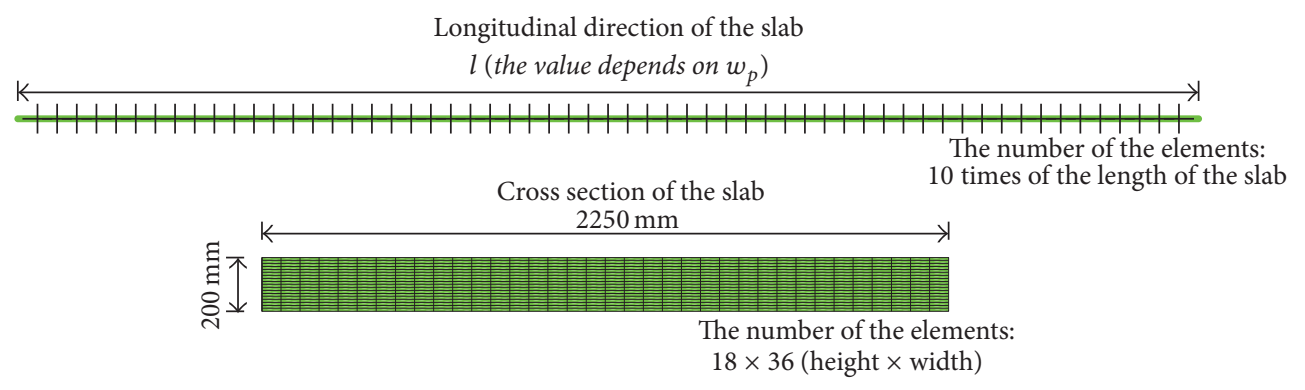

Figure 9: Mesh density of the slab.

the deformation remains unchanged, the longer the halfwavelength develops, the less the energy associated with bending the slab is and also the more the gravitational potential energy of track slab is, and vice versa. Therefore, it can be inferred that there must be a most unfavorable half-wavelength of deformation $l_{\text {min }}$ which corresponds to a minimum of the temperature force. According to the definition of functional derivation, the half-wavelength could be obtained by

$$
\frac{\partial F(l, w)}{\partial l}=0
$$

and hence

$$
l_{\min }=\sqrt[4]{\frac{K E I \pi^{5}\left(w+w_{e}\right)}{4 \rho A g}} .
$$

The upwarping deformation has a maximum value with respect to the rising of temperature force. The following formula is obtained by taking derivative of temperature force $F$ as a stationary value:

$$
\frac{\partial F(l, w)}{\partial w}=0
$$

We can derive

$$
l_{w}=\sqrt[4]{\frac{K E I \pi^{5} w_{p}}{4 \rho A g}} .
$$

When $l_{w}=l_{\min }$, the relationship among the amplitude of the deflection of the coupled slabs, the amplitude of the initial elastic misalignment, and the amplitude of the initial plastic misalignment is $w_{p}=w+w_{e}$. Since the parameters $w_{p}$ and $w_{e}$ are variable, we assume that $w=w_{e}$ to a certain condition. Once $w$ is given, the other two parameters $w_{p}$ and $w_{e}$ can be determined. Substituting the above conditions $\left(l=l_{\min }, w=\right.$ $w_{e}$, and $w_{p}=w+w_{e}$ ) in (20), if the temperature force $F$ is taken as a stationary value, then we have

$$
F=\frac{\sqrt{2 K E I \pi^{5} w \rho A g}}{4 \pi^{3} w} .
$$

\section{Formula Verification and Parametric Study}

The primary objective of this section is to quantify the upwarping in coupled slabs and determine its effect on the bending behavior of the coupled slab. Finite element method was utilized to verify whether the analytical solution correlates with the results of the FEM. As mentioned previously from the survey at Ningbo-Hangzhou High-Speed Railway in 2013, the maximum value of the amplitude of the total deformation $w_{p}+w+w_{e}$ may reach $20 \mathrm{~mm}$. Since we have such a relationship, $w_{p}=w+w_{e}$, we can find that $2 w_{p}=$ $20 \mathrm{~mm}$, so $w_{p}=10 \mathrm{~mm}$. On the other hand, the initial plastic misalignment is caused by the coupled effect of the manufacture error, creep, and thermal expansion of concrete, and such a misalignment in the coupled slabs holds the same reasons as the CWR. Since these adverse effects have a greater influence on the coupled slabs and the amplitude of the initial plastic misalignment in CWR is $3 \mathrm{~mm}$ [15], we calculate the temperature force with the amplitude of the initial plastic misalignment in LCPST starting from $4 \mathrm{~mm}$. Therefore, the amplitude of the initial plastic misalignment $w_{p}$ in this section ranges from $4 \mathrm{~mm}$ to $10 \mathrm{~mm}$.

3.1. Model and Parameters in ANSYS. The slab with liner density of $2500 \mathrm{~kg} / \mathrm{m}^{3}$ is applied and simulated by the continuously supported Euler beam. The element type of beam 188 in the finite element model is adopted in ANSYS software, which is suitable for analyzing slender to moderately stubby/thick beam structures. The element is based on Timoshenko beam theory which is a first-order shear-deformation theory: transverse-shear strain is constant through the cross section. This element uses the slenderness ratio of a beam structure $\left(G A l^{2} /(E I)\right)$ to judge the applicability of the element, where $G$ is shear modulus; it can take a value between $E / 3$ and $E / 2$, and other parameters are mentioned previously. A slenderness ratio greater than 30 is recommended; our model's slenderness ratio is 416.025 , far more than 30 , so it is suitable for simulating the slab. As shown in Figure 9, the mesh density of the slab in length is 10 times of the slab length, and those in the height and width are 18 and 36, respectively. Thus, the total number of elements used in the FE model is $10 \times l$ (length of the model) $\times 18 \times 36$. The nonlinear spring is used to simulate the supporting between the slab and CA mortar. COMBIN39 is a unidirectional element with nonlinear generalized forcedeflection capability that can be used in any analysis. So the 
TABLE 2: Comparison of vertical deformation $w$ between analytical solution and the result of the FEM.

\begin{tabular}{lccccc}
\hline Number & $w_{e} / \mathrm{m}$ & $w_{p} / \mathrm{m}$ & $\begin{array}{c}\text { Analytical } \\
\text { solution } \\
w / \mathrm{m}\end{array}$ & $\begin{array}{c}\text { ANSYS } \\
w / \mathrm{m}\end{array}$ & $\begin{array}{c}\text { Relative error } \\
1\end{array}$ \\
\hline & 0.002 & 0.004 & 0.002 & 0.001995 & $0.25 \%$ \\
3 & 0.0025 & 0.005 & 0.0025 & 0.002525 & $0.73 \%$ \\
4 & 0.003 & 0.006 & 0.003 & 0.003022 & $0.77 \%$ \\
5 & 0.0035 & 0.007 & 0.0035 & 0.003473 & $0.2 \%$ \\
6 & 0.004 & 0.008 & 0.004 & 0.003992 & $2.6 \%$ \\
7 & 0.0045 & 0.009 & 0.0045 & 0.004383 & $2.64 \%$ \\
\hline
\end{tabular}

supporting of CA mortar can be simulated with COMBIN39 spring element, with the thickness of $0.03 \mathrm{~m}$.

The elastic modulus of the supporting of CA mortar with $8,000 \mathrm{MPa}$ [19] is applied in the calculation model. The supporting area of CA mortar is equal to the bottom of $l \times 2.55$ (slab width) $\mathrm{m}^{2}$. With the number of divided elements of track slab $N$, the stiffness of the spring $k$ yields

$$
k=\frac{8 E^{9} \times l \times 2.55}{0.03 \times(N+1)} .
$$

Hence, the relationship between the supporting force of the spring and displacement $y$ is

$$
F_{k}= \begin{cases}k \cdot y=\frac{8 E^{9} \times l \times 2.55}{0.03 \times(N+1)} y, & y<0, \\ 0, & y \geq 0\end{cases}
$$

where $F_{k}$ is the interaction force between the supporting of the CA mortar and the coupled slab and $y$ is the relative displacement between the supporting of the CA mortar and the coupled slab, which is negative when the spring is compressed. The lower end of spring is applied with full restraint, as displayed in Figure 10.

The elastic modulus of prefabricated slab $E$ is taken as $3,5500 \mathrm{MPa}$, and the thickness and width of slab are taken as $0.2 \mathrm{~m}$ and $2.55 \mathrm{~m}$, respectively, and the coefficient of linear expansion $\alpha$ is $10^{-5}$. The calculation model is shown in Figure 10, in which the slabs are subject to both the effect of temperature and the dead weight.

3.2. Formula Verification. With the initial conditions of $w_{e}=$ $2 \mathrm{~mm}, w_{p}=4 \mathrm{~mm}$, and $w=2 \mathrm{~mm}$, the most unfavorable half-wavelength of the deformation $l_{\min }$ of $6.18 \mathrm{~m}$ is calculated out according to (22) or (24). And we can also get the temperature force $F(15,383.377 \mathrm{kN})$ by using $(25)$; the value of the temperature force is converted to temperature and then applied in the finite element model with the initial misalignments of $y_{e}+y_{p}$. According to the comparison between the obtained vertical maximum displacement $(y=$ $1.995 \mathrm{~mm}$ ) and the given parameter $w$ in the analytical solution, the relative error is only $0.25 \%$. From the compared results displayed in Table 2, we can obtain the fact that the

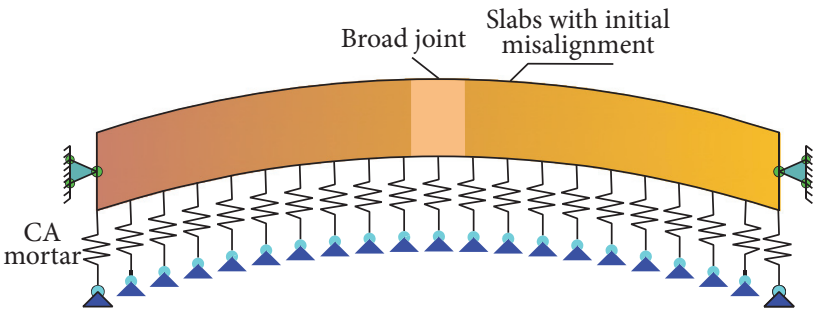

FIGURE 10: Finite element model for the coupled slabs with initial misalignments in ANSYS.

calculation result of derivation formula is in accordance with the calculation result of ANSYS. Figure 11 shows the contour plots of ANSYS calculation results.

3.3. Parametric Study. The presented model was used to perform a parametric study and to evaluate the influence of the considered parameters on the upwarping vertical deformation of LCPST. The study in this section analyzed the effect of the amplitude of the initial elastic misalignment $w_{e}$, the amplitude of the initial plastic misalignment $w_{p}$, the amplitude of the deformation of the slabs $w$, and the most unfavorable half-wavelength $l_{\min }$.

It could be seen from Table 2 that the relative error between parameter $w$ for formula calculation and deformation output of finite element method is relatively small. In Table 2 , the changes of the parameters $\left(w_{e}, w_{p}\right)$ do not vary in random, they affect each other, and $l_{\min }$ is the value with respect to $w_{p}$. In general, they represent a series of peak values, which consist in a series of discrete points, which indicates the deformation of track slab, and other parameters, $w_{e}, w_{p}$, and $l_{\min }$, in the formula are not independent, and the deformation of the upwarping is getting larger along with the increase of initial misalignments.

Figure 12 and (24) show that the most unfavorable half-wavelength $l_{\min }$ is closely related to the amplitude of the initial plastic misalignment. The most unfavorable halfwavelength of deformation increases with the increase of initial misalignments. Since the upwarping generally occurs at broad joints, the length of one longitudinal coupled slab is $6.45 \mathrm{~m}$ and the calculated longest half-wavelength is $7.769 \mathrm{~m}$, 


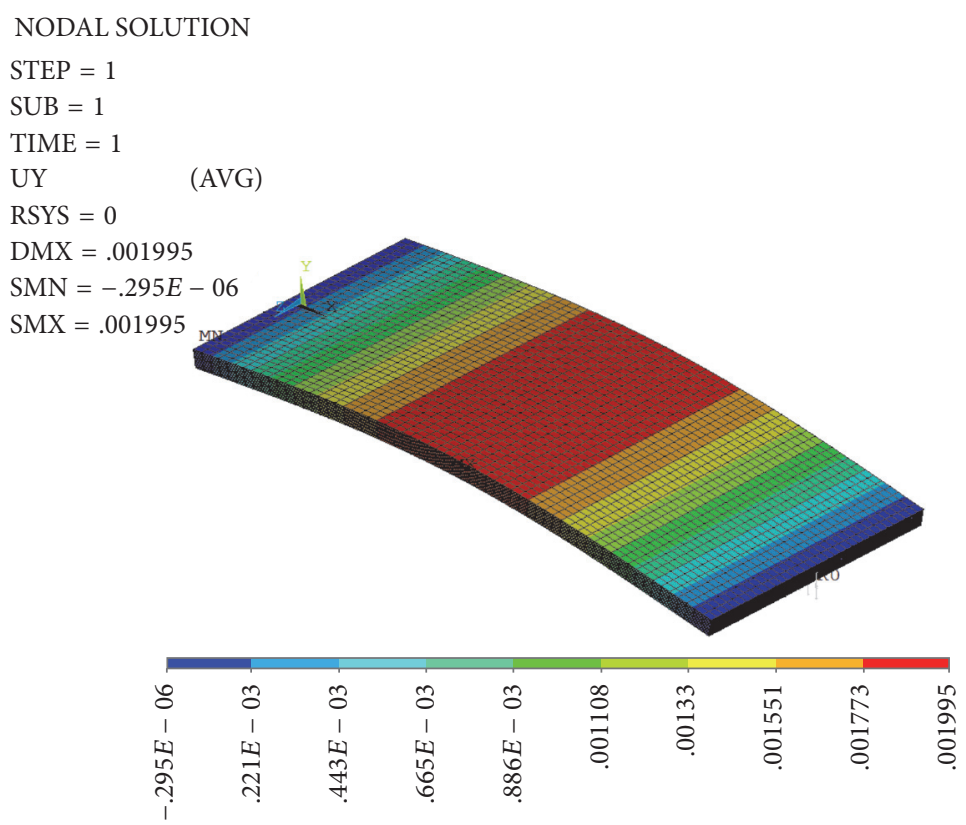

(a)

\section{NODAL SOLUTION}

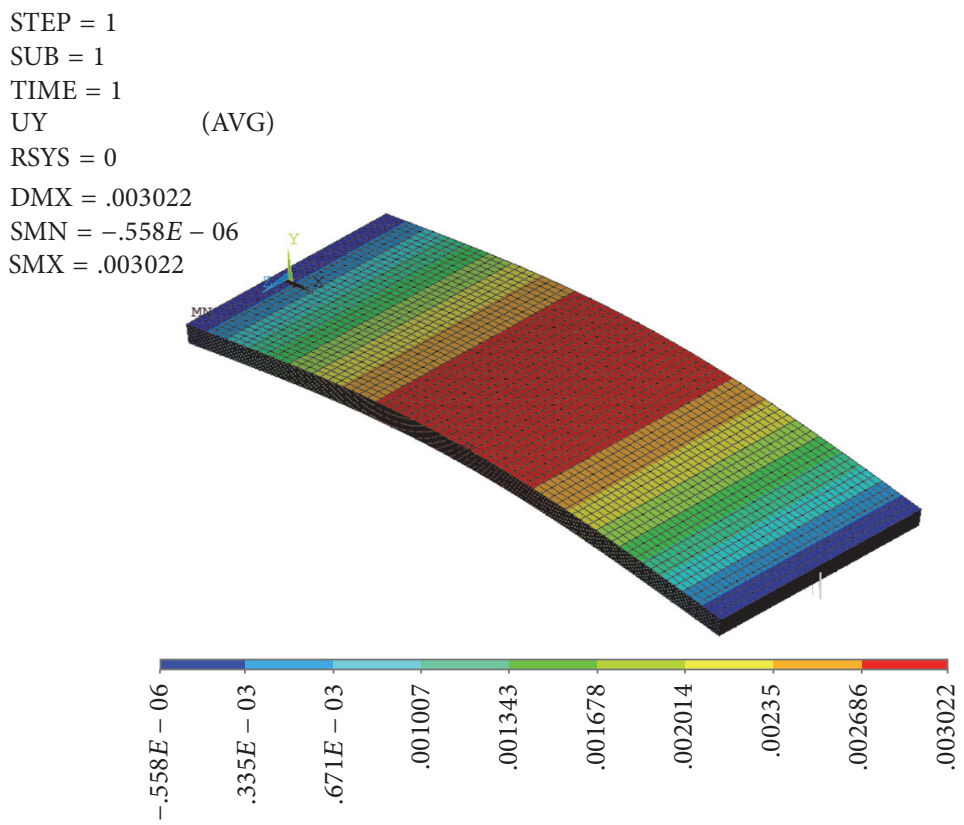

(b)

FIGURE 11: Contour plot of vertical displacement of the slabs in the ANSYS model ((a) with $w_{e}=2 \mathrm{~mm}, w_{p}=4 \mathrm{~mm}$, and $l_{\min }=6.2 \mathrm{~m}$ and (b) with $w_{e}=3 \mathrm{~mm}, w_{p}=6 \mathrm{~mm}$, and $l_{\min }=6.8 \mathrm{~m}$ ).

which is no more than the length of 2 slabs; hence, the influence of adjacent broad joints could be ignored.

It could be seen from Table 3 that when the amplitude of the initial elastic misalignment $w_{e}$ is $2 \mathrm{~mm}$ and initial plastic misalignment's $w_{p}$ is $4 \mathrm{~mm}$, the most unfavorable halfwavelength $l$ equals $6.18 \mathrm{~m}$; the temperature force necessary for upwarping of the track slab up to $2 \mathrm{~mm}$ is $15383.377 \mathrm{kN}$; when $w_{e}$ is $5 \mathrm{~mm}$ and $w_{p}$ is $10 \mathrm{~mm}, l$ equals $7.77 \mathrm{~m}$; the temperature force necessary for upwarping of the track slab up to $5 \mathrm{~mm}$ is $9729.302 \mathrm{kN}$, which illustrates that the more terrible the initial misalignments defect of track slab is, the longer the most unfavorable half-wavelength is and also the more unstable the track slab is; that is, unwarping is more likely to occur. Therefore, it is recommended to reduce the initial misalignment caused by the coupled effects of creep, thermal expansion, and manufacture error of track slabs to 


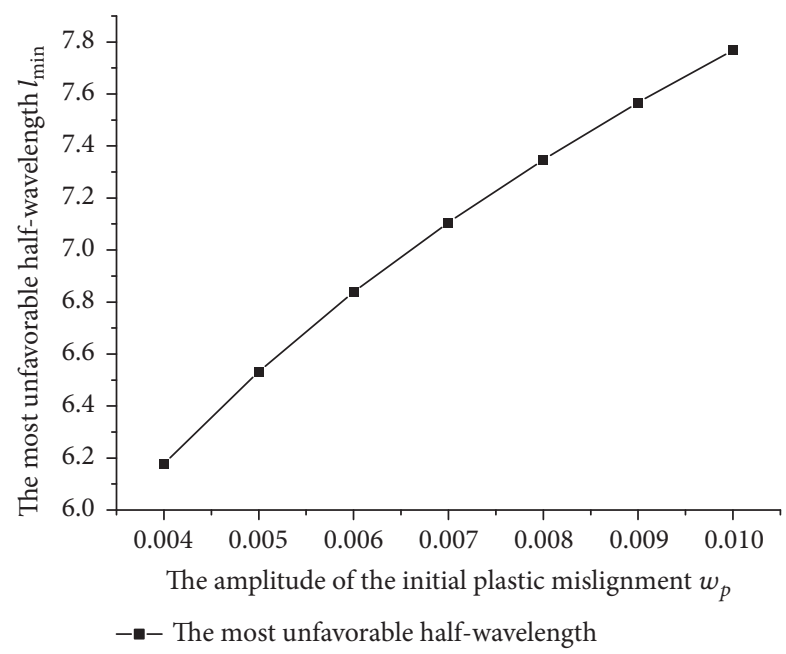

FIGURE 12: Relationship between the amplitude of the initial plastic misalignment and the most unfavorable half-wavelength of deformation.

TABLE 3: Relationship among the amplitude of the initial misalignments, most unfavorable half-wavelength, and temperature force.

\begin{tabular}{lccccc}
\hline Number & $w / \mathrm{m}$ & $w_{e} / \mathrm{m}$ & $w_{p} / \mathrm{m}$ & $l / \mathrm{m}$ & $F / \mathrm{N}$ \\
\hline 1 & 0.002 & 0.002 & 0.004 & 6.178492 & 15383377 \\
2 & 0.0025 & 0.0025 & 0.005 & 6.53296 & 13759310 \\
3 & 0.003 & 0.003 & 0.006 & 6.837626 & 12560475 \\
4 & 0.0035 & 0.0035 & 0.007 & 7.106275 & 11628740 \\
5 & 0.004 & 0.004 & 0.008 & 7.347507 & 10877690 \\
6 & 0.0045 & 0.0045 & 0.009 & 7.567077 & 10255585 \\
7 & 0.005 & 0.005 & 0.01 & 7.769043 & 9729302 \\
\hline
\end{tabular}

prevent the coupled slabs from large upwarping within a smaller temperature force.

\section{Conclusions}

In this paper, a study on the effect of upwarping on the longitudinally coupled prefabricated slab track was conducted by establishing an analytical expression using energy method which applies energy method to quantify the vertical upwarping deformation of the coupled slabs. The main contribution of the expression is that it can quantify the vertical upwarping of the coupled slabs with both of the gravity of the slabs and the initial misalignments of the slabs taken into account. Then a finite element model of the coupled slabs for the upwarping was built to demonstrate the analytical solution's adequacy. The analytical solution may accurately predict the observed behavior of the presented problem. Based on the limited results in this study, several conclusions can be drawn as follows:

(1) Based on the results between the analytical solution and the solution in FEM, it can be concluded that when the amplitude of the initial elastic misalignment $w_{e}$ is equal to a half of the amplitude of the initial plastic misalignment $w_{p}$, the relative error between the deformation parameters from formula calculation and deformation output from FEM is relatively small (the maximum value of the relative error is $2.64 \%$ ). The response predictions from the analytical solution correlate well with the solution in FEM.

(2) The most unfavorable half-wavelength of the deformation $l_{\min }$ is closely related with the amplitude of the initial plastic misalignment $w_{p}$. With the increase of the amplitude $w_{p}$, the most unfavorable half-wavelength $l_{\min }$ becomes longer. The calculated maximum half-wavelength is $7.769 \mathrm{~m}$, thus less than the length of 2 slabs (the length of LCPST is $6.45 \mathrm{~m}$ ), so the influence of adjacent broad joints could be ignored, which is in accordance with assumption (e) in Section 2.2.

(3) When the amplitude of the initial misalignments $w_{e}$ $+w_{p}$ exaggerates, the vertical deformation of the slabs will be amplified with a smaller temperature force than that of the slab with smaller initial misalignments; namely, the slab with larger initial misalignments will be much likely to suffer upwarping. It is thus recommended to reduce the initial plastic misalignment caused by the coupled effects of creep, manufacture error, and thermal expansion of the coupled slabs to prevent the coupled slabs from large upwarping within a smaller temperature force.

\section{Nomenclature}

$y_{f}: \quad$ Vertical deformation of the coupled slabs caused by temperature force

$w$ : Amplitude of the upwarping deformation

$l$ : Half-wavelength of the slabs

$y_{e}: \quad$ Initial elastic misalignment

$w_{e}: \quad$ Amplitude of the initial elastic misalignment

$y_{p}: \quad$ Initial plastic misalignment

$w_{p}: \quad$ Amplitude of the initial plastic misalignment

$R: \quad$ Radius of vertical curve

$y_{R p}$ : Initial plastic misalignment caused by vertical curve

$y_{\text {Ftotal }}$ : Total deformation curve of LCPST with temperature force $\left(y_{f}+y_{e}+y_{p}+y_{R p}\right)$

$y_{\text {ototal }}:$ Total deformation curve of LCPST without temperature force $\left(y_{e}+y_{p}+y_{R p}\right)$

$F: \quad$ Compressive temperature load

$W: \quad$ Total potential energy of the slabs

$W_{1}$ : Deformation energy associated with compressing the slabs

$W_{2}$ : $\quad$ Energy associated with bending the slabs

$W_{3}: \quad$ Gravitational potential energy of the slabs

A: $\quad$ Cross section area of the slabs

$\Delta: \quad$ Change in the arc length (not the chord length) of the coupled slabs 
$\Delta l_{F}:$ Arc-chord difference after deformation

$\Delta l_{0}$ : Arc-chord difference before deformation

$d s_{0}$ : Arc-differential before deformation

$d s_{F}$ : Arc-differential after deformation

$M$ : Internal bending moment during the process of deformation

$M_{e}$ : Internal bending moment of the initial elastic misalignment

$\theta$ : Angle of rotation induced by the upwarping of the slabs

$E I$ : Flexural rigidity of the slab

$E_{1} I_{1}$ : Flexural rigidity of broad joint

$q$ : Force per unit length generated by the gravity of coupled slabs

$\Delta l$ : Length of broad joint

$K$ : Reduction factor of flexural rigidity of track slab due to the influence of dummy joints

$F_{k}$ : The interaction force between the supporting of the CA mortar and the coupled slab.

\section{Conflicts of Interest}

The authors declare that there are no conflicts of interest regarding the publication of this paper.

\section{Authors' Contributions}

Xueyi Liu, Zhibin Jin, and Juanjuan Ren conceived the theory; Junbin Yang and Shijie Deng performed the theory; Juanjuan Ren built the slab model and analyzed the data; Juanjuan Ren, Shijie Deng, and Zhibin Jin wrote the paper.

\section{Acknowledgments}

This research is sponsored by the National Natural Science Foundation of China (nos. U1434208 and 51578472) and China Railway Corporation (no. 2015G001-F) whose support is gratefully acknowledged. The useful contribution and discussions from project partners are also acknowledged.

\section{References}

[1] X. Liu, P. Zhao, R. Yang, and P. Wang, Design Theory and Method of Ballastless Track of Passenger Dedicated Railways, Southwest Jiaotong University Press, Chengdu, China, 2010.

[2] B. Lichtberger, Track Compendium: Formation, Permanent Way, Maintenance, Economics, Eurail Press, 2005.

[3] J. Ren, R. Yang, P. Wang, P. Yong, and C. Wen, "Slab upwarping of twin-block slab track on subgrade-Bridge transition section-Parameter Study and Repair Method," Transportation Research Record, vol. 2448, pp. 115-124, 2014.

[4] Y. Liu and J. Feng, "Treatment of upheaval of CRTS IItype ballastless track slab for Beijing-Tianjin intercity railway," Railway Engineering, vol. 2, pp. 142-145, 2016.

[5] F. Liu, Z. Zeng, and B. Wu, "Research on the CRTS II slab ballastless track buckling deformation in the process of construction," Journal of Railway Engineering Society, vol. 32, no. 1, pp. 55-60, 2015.
[6] H. Lin, X. Liu, and R. Yang, "Compressive stability of continuous-slab-track on long-span bridge," Xinan Jiaotong Daxue Xuebao/Journal of Southwest Jiaotong University, vol. 43, no. 5, pp. 673-678, 2008.

[7] Z. Zhang, Z. Zeng, F. Liu, Y. Zhang, and D. Zeng, "Research on the longitudinal displacement between track plate and filling layer under the influence of temperature," Advanced Materials Research, vol. 906, pp. 323-328, 2014.

[8] Q. Xu and X. Zhang, "Longitudinal forces characteristic of Bogl longitudinal connected ballastless track on high-speed railway bridge," Zhongnan Daxue Xuebao (Ziran Kexue Ban)/Journal of Central South University (Science and Technology), vol. 40, no. 2, pp. 526-532, 2009.

[9] A. D. Kerr, "Analysis of thermal track buckling in the lateral plane," Acta Mechanica, vol. 30, no. 1-2, pp. 17-50, 1978.

[10] I. N. Martínez, I. V. Sanchis, P. M. Fernández, and R. I. Franco, "Analytical model for predicting the buckling load of continuous welded rail tracks," Proceedings of the Institution of Mechanical Engineers, Part F: Journal of Rail and Rapid Transit, vol. 229, no. 5, pp. 542-552, 2015.

[11] Y. Luo, L. Li, and H. Yin, "A dynamic analysis of a continuous welded rail track under a longitudinal stress caused by temperature changes," Proceedings of the Institution of Mechanical Engineers, Part F: Journal of Rail and Rapid Transit, vol. 224, no. 2, pp. 91-101, 2010.

[12] A. Manap, "Mathematical modeling for longitudinal displacement of a straight continuously welded railway track and its numerical implementation," Key Engineering Materials, vol. 462-463, pp. 801-806, 2011.

[13] S. Xu, The Application of the Variational Principle in the Stability Calculation of CWR, China Railway Publishing House, Beijing, China, 1985.

[14] China Railway Fourth Survey and Design Institute Group Co. Ltd., Code for Design of Railway Continuous Welded Rail, 2012.

[15] J. Ren, Railway Track Technology, Southwest Jiaotong University Press, 2014.

[16] J. Liu and Z. Guang, "Stability calculation for continuous welded tracks and selection of the temperature deformation curve form function," Journal of the China Railway Society, vol. 9, pp. 62-73, 1987.

[17] B. Bai, "Design standard of longitudinal section of high speed railway and its application," Railway Standard Design, vol. 7, pp. $4-7,2010$.

[18] “Code for Design of High Speed Railway,” TB 10621-2014, China Railway Press, 2014.

[19] China Academy of Railway Sciences, Central South University, Tsinghua University, and China Petroleum Chemical Co Shanghai Asphalt Branch, Interim Technical Requirement for Cement Emulsified Asphalt Mortar of the CRTS II-Type Slab Track in Passenger Dedicated Line, 2008. 


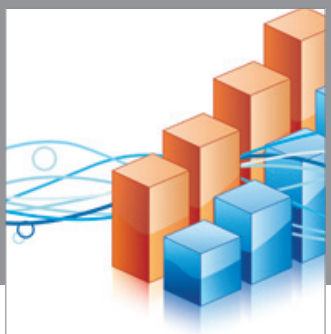

Advances in

Operations Research

vatem alat4

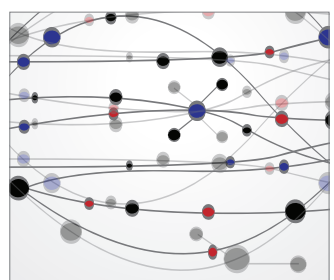

\section{The Scientific} World Journal
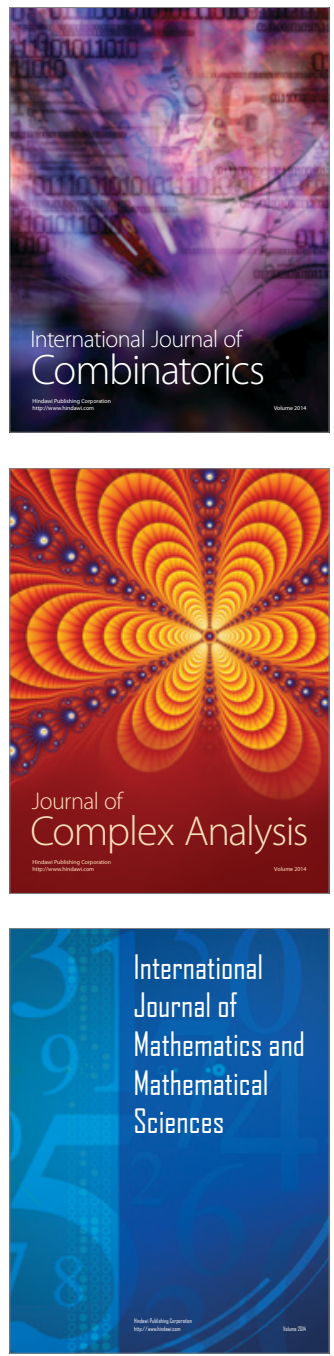
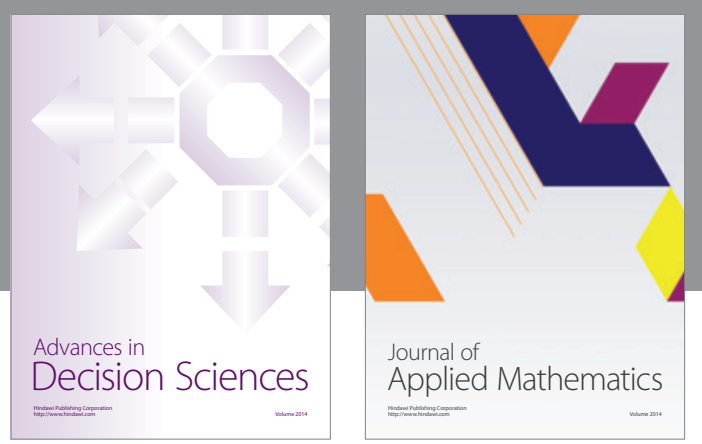

Algebra

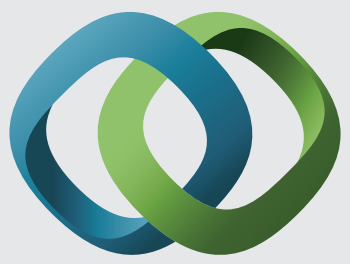

\section{Hindawi}

Submit your manuscripts at

https://www.hindawi.com
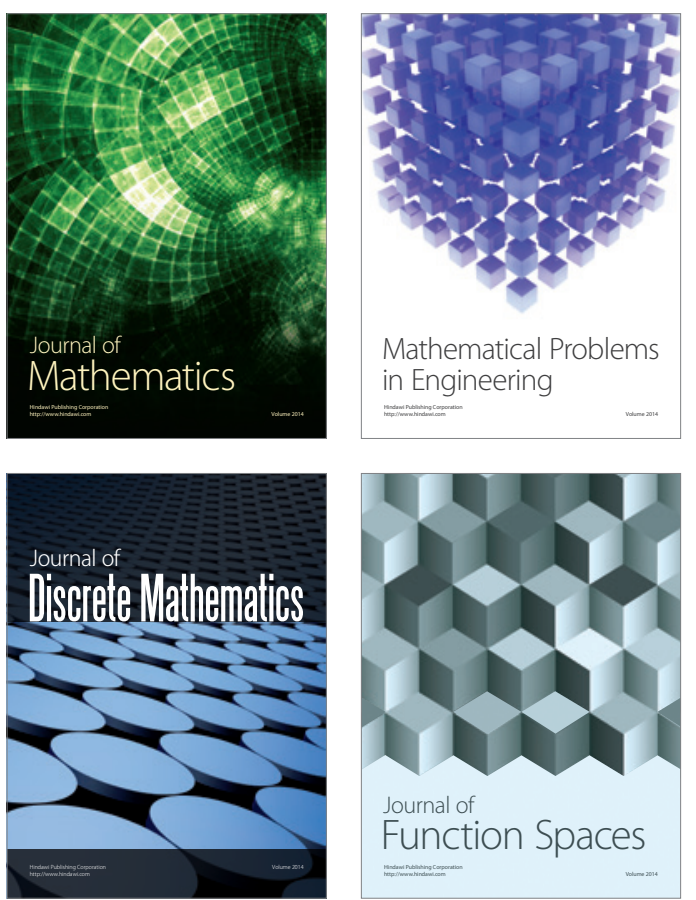

Mathematical Problems in Engineering
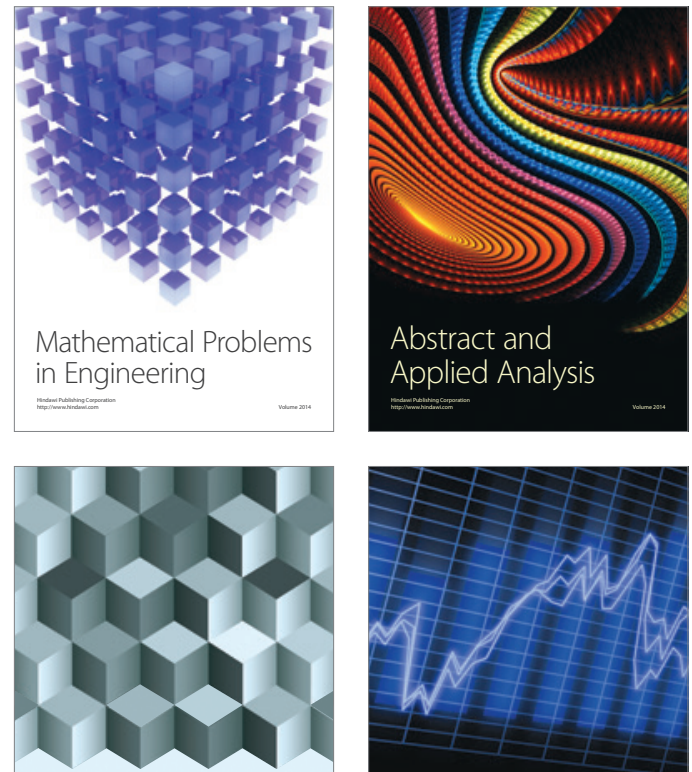

Journal of

Function Spaces

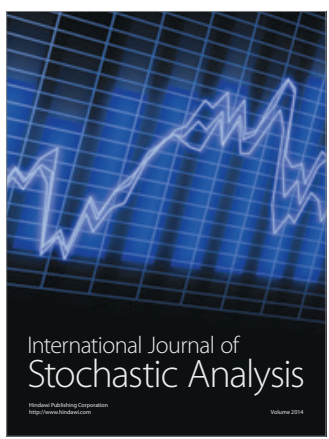

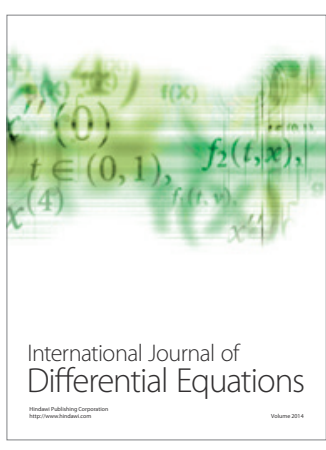
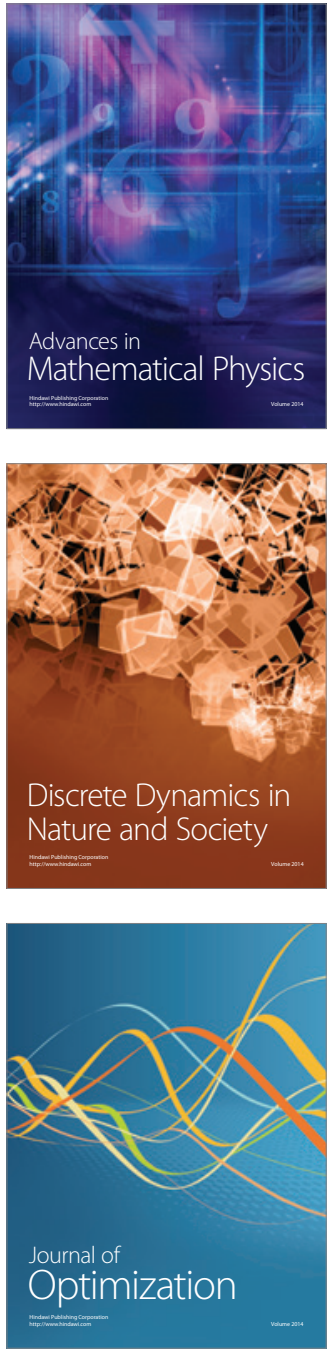